\title{
Research of Visual Performance in Visual Communication Design
}

\author{
Bo Liu \\ Ningxia Normal University, Yinchuan, Ningxia, 756000
}

Keywords: visual communication design; visual space; graphics; new media

\begin{abstract}
With the development of society, the visual space is more and more effective in our lives. The existence of visual space plays an important role in visual communication. Through the visual dimension, the overall effect of vision is presented in our minds, and visual design is established based on visual perception. The visual cognition needs to have a certain order, and can be performed well through the design. In this process, visual communication is designed to make the vision more three-dimensional with more sense of design. The presentation and design of visual space is based on visual cognition and in-depth information. The way in which visual communication is generally presented is mainly through visual design of graphics, colors, and so on. With the development of network technology and the blending of new media forms, diversity in its visual communication has changed. Visual communication is made quickly and profoundly based on visual information. Through the expression of graphics, colors, and words, the forms of expression are varied and the methods of expression are different. Through the multi-faceted development of visual design, the visual space has a more meaningful meaning. In this process, the development of the information age is combined to enhance the development of the visual space and its research is of great significance. This paper is mainly from the perspective of visual communication, the concept of visual space is elaborated, and visual performance in the design of visual communication is studied, so as to put forward some suggestions and explore the current trend of the integration of visual aspects with new media.
\end{abstract}

\section{Introduction}

With the development of society, visual design increasingly acts on our material life. While enjoying material life, visual design can meet the aesthetic requirements and satisfy people's perception of things. Especially in designing so many things now, the requirements of design can not only satisfy the current state, but also need to continuously excavate and innovate design elements, satisfy people's increasing appreciation level, and have higher and higher requirements for designers. People are no longer satisfied with a single design element, and they need a more interactive experience in the real space, satisfying people's interests from the visual space aspect, and based on this, visual communication design is represented. With the development of science and technology, visual communication design is playing a more and more important role in the visual space. It will make information more quickly visual experience.

\section{The Overview of Visual Space}

\subsection{The content of visual space}

The performance of the visual space is the spatial range that we can see with the eyes. In this range, the spatial structure is combed, and the spatial structure is considered from the top to the bottom, and from the left to the right visual space, through the spatial position. The internal and external gazes we see interact with each other. From our point of view as the starting point, the confirmation of the relationship between length, width and height in space will become the main manifestation of visual space. In this process, information is constantly being transmitted, visual associations caused by images, visual spaces, etc., and the visual space in the communication can be carried out by the transmission of information, the transmission of the gaze, and the real space transmission, and the visual space can be carried out. expression. 


\subsection{The construction of visual space}

The visual space is a very large space for designers, and the construction of the space is mainly based on the structures in the line of sight that we see as the starting point. When our eyes are focused on the same focus, time and space will all gather in this focus area, so we will present a clearer effect. Through a series of backgrounds outside this focus, we will become a foil. When we look at the background, At the moment, these are still vague. Therefore, the clarity and ambiguity of the line of sight are determined by our eyes. The eyes are the windows of the mind. Through the eyes, we can see a lot of things that are visually conveyed. By watching the focus, we will pay attention to the visual selection, and the attention will be clearer. And scattered around it will be relatively vague. When we look at a chair in the visual space, a book is placed on the chair and the chair is partially blocked. We see a complete chair, but in this space, a book holds a part of the chair. To shelter, it was only in our eyes that the chair was blocked. But our perception is very clear. In this space, the chair is still a complete chair. The book is also a complete book, but it is only covered. In life, the objects we see often start from a single point of focus, perceiving their unobstructed parts. For example, when we look at the chair just now, it is clear that the chair is a three-dimensional, but we are very clear in the observation of such a three-dimensional items, but through the naked eye we may see a corner of the chair or One leg, but the side that is hidden or invisible by the line of sight is also true, so in the process of cognition, by understanding, taking our gaze point as the center point and expressing the coordinates in space, it will be divided into one The overall dimension, this dimension can be expressed in terms of up and down, left and right, and depth.

\subsection{Methods to express visual space}

We live in a three-dimensional space where activities are carried out. Through this space, we acquire information. From our own perspective, we can observe things in the space. We can change the goal of gaze through the direction and angle of gaze. The transmission of information conveys the content to our brains. Through the transmission of information, the perception space can be quickly recognized, and the space can be clearly seen by both eyes. This is because most of the objects can only be recognized and cannot be seen. Therefore, the brain is performing The time of analysis is based on the observation of the eye. If you conduct more in-depth information, it will be more difficult to control. The perception of the eyes is usually different to some degree. When the plane pattern is perceived, the brain's judgment is relatively shallow, which is relative to the three-dimensional space, in-depth exploration In the process, the distance between the objects needs to be processed. In different perceptions, the perception is not the same. Different depths also reflect different motion parallaxes. This will block or shade the objects we see. . In the consideration of perception space, some fragmented graphics and clues in the brain need to be dealt with in depth, allowing designers to break through their different thinking spaces to communicate visually, and to present this combination of methods through set goals. Really patterned surfaces perform specific effects and enhance cost comparisons.

\section{The Main Design of Visual Space in Visual Communication}

\subsection{The effect of graphic language in visual communication}

The visual communication has a certain history. In the 1940s, scholars in the United States elaborated the main points of visual communication. The main process is to transfer information to each other and pass it through the human visual sense. This kind of language process that makes the recipient feel good and will produce visual understanding is the process of transferring the substance. This process is to receive The person brings a good visual sense. The role of graphics in visual communication is the most recognizable. By using graphics and artistic processing to integrate graphics into design works, graphics can be depicted on the plane. In the design of graphics, the perception of graphics can be performed through the brain. Visual operation. Especially in the initial life, the ability to perceive graphics exceeds numbers. In the process of 
cognition, graphics have language capabilities. People have more profound definitions of graphics, and in the process of processing new graphics language, the cognitive time of the brain is relatively early. In this process, the vision needs to be changed and the simple graphic language is overdrawn to a more complex graphic language. This is actually different from the experience, and many graphic languages are ours. As we all know, for example, the cups of drinking water that we often come into contact with in daily life, the desks used, and so on, the graphic language in western Zhejiang forms a certain impression in the mind through visual communication, and can be successfully identified and strengthened through visual thinking. Attractiveness allows it to be used and understood in the process of gazing and to clarify the role of the graphical language.

\subsection{The role of graphical language in the visual space for visual communication design}

In the process of visual communication, judgment is often made through the recognition of graphics. However, the graphics are malleable. When extending from the plane graphics to the three-dimensional graphics, part of the visual illusion will appear. The contour of the visual illusion can be divided into Colors and graphic outlines and more. The illusion of the outline of the figure is mainly from the motion of one point to the multiple lines. The line has horizontal and vertical lines. In the process of visual movement, because the point of fixation is constantly moving, the whole picture will follow. If you move, you will have a visual illusion. The graphical language has a deeper impression in the mind, and combines the centralized graphics to extend the center image. The scattered points around it can also be extended. The surrounding of the image is also expanded to form a relatively deep space. Arranging graphics with different shapes has a certain effect on visual communication.

\subsection{Language performance of visual space in color}

In our daily life, our perception of color is very profound. Color also has an important effect on our mood. For example, in hot weather, red is too hot and black is too hot. Deeper understanding, but if you change to white, the relative feeling is refreshing and so on, the role of color in the brightness and purity of the color will give us a different visual experience, therefore, the use of color in the visual communication plays an important role effect. For example, the red five-star red flag reminds us of the country. When we see lemon, it makes us think of saliva. The feeling reflected in the color may even affect thinking and senses. People who have different feelings about color will have different psychological feelings. Different mental associations will also be produced. In the visual space, visual language will be used to convey visual effects, which is of crucial importance in the expressiveness of the visual space. In life, people who like sports generally wear brightly colored clothes. This has a great impact on vision, stimulates movement and increases attention. Especially in a relatively new environment, many people mainly pay attention to their colors. In the space where we live, when we carry out interior decoration, we always pick the color we like. This color is in line with the innermost color scheme. Physical and psychological pleasure. Usually color can be collocated, this combination will have a root effect, the design of the color will be based on the pattern of color, the tone will be adjusted as a whole, etc. Different colors create different space, in this relationship, Brighter colors and warm colors will give people different feelings, and will also have a visual impact on the size and volume of the space. While performing color modeling, people's feelings are also different, so consider the color The role of space effects, the color language in the visual space.

\section{The Development of Visual Space under the Influence of New Media}

\subsection{Relations of visual communication design and new media}

The influence of the development of new media on visual space is relatively large. The appearance of human-computer interaction system under the effect of visual communication influences the development of visual space. With the continuous advancement of science and technology, computer networks highlight their advantages in applications. Under the influence of 
new media, the design and experience of human interactive interfaces needs continuous research, from the application of two-dimensional code payment terminals to touch-based experience. Management and visual communication effects are very necessary for the development of new media. At the moment, they need to be visually communicated to the audience for different experiences and feelings.

\subsection{The role of computer technology in visual communication design}

The design of visual communication technology requires a certain amount of space for the application of the computer. When carrying out the interface design, computer support is needed to create a better sense of space. It is also necessary to construct a virtual space, imitate the real space through the simulated space, process the real logic, and control the operation and commands of the system and the interface design basis of the human-computer interaction through the computer program, and execute the data command. Formulation, through the control of the thinking mahjong to present the two-dimensional world, so put the computer into the visual communication will be reflected in our design based on humane design.

\section{The important manifestation of visual space in visual communication}

In the process of visual communication, it enters our eyes with the visual experience of two-dimensional space. Based on this, we perceive the three-dimensional space, and in the processing of space, the perception ability influences the visual expression, and in the visual communication. The full recognition of graphic information and color text information is conducive to the performance of visual space. The design of new media brings more room for development to the visual space. In the application of new media, the virtual sense of the visual space and the sense of innovation need to be conveyed through visual communication to make people feel real, in order to meet people's electronicity. And the mechanized experience will give full play to the visual space to promote. Especially in the design, a reasonable visual space is very important for the communication of information. This requires the transmission of information content and the presentation of visual effects. This requires the analysis of visual spatial effects and the introduction of cognitive sensations to people. Perceived space. Among them, the resolution of the aesthetic space is the most important. The aesthetics in the visual space are excavated by certain rules, and the color, lines, etc. are transmitted through the information of the beauty, allowing people to perceive the real space. This is the visual space for aesthetics. Requirements.

\section{Conclusion}

It can be seen from the visual space in visual communication that in the expression of new media, visual communication is conveyed in the aesthetics, the graphic language and the color language in the visual thinking space through different forms of visual space. Constitute a certain visual presentation, there is also a certain value for aesthetics.

\section{References}

[1] Wan Zehui. Visual space research based on visual information communication art angle[J]. Wireless Internet Science and Technology, 2016, 01:124-126.

[2] Xu Ling. Innovative Deconstruction and Reconstruction of Textual Design in Visual Communication Design [J]. Art, 2016, 2902:44.

[3] Fan Xiaoyi, Fan Xiaoqi, Yu Dinghong. Visual Communication Design of Online Courses[J]. Journal of Tonghua Teachers College, 2011, 3202:77-80.

[4] Chai Zhi. Research on visual space transformation in graphic design [J]. Art and Design (Theory), 2011, 209:62-63. 
[5] Chen Jie. New development of visual communication design in space design [D]. Tianjin University of Science and Technology, 2015.

[6] Fan Wei. Space Situation Construction in Visual Communication Design [D]. Hubei University of Technology, 2010.

[7] Zou Xianxiang. Emotional factors and expression in visual design [J]. Qunwen Tiandi, 2012, 04: 275-277.

[8] Huang Guanglie. Research on the role of graphic design in indoor space design [J]. Modern Decoration (Theory), 2012, 08: 44+46.

[9] Cao Wenqi. Innovation of visual thinking mode in visual communication design [J]. Jiangxi Social Sciences, 2012, 3208:216-219.

[10] Wei Yang. Application of graphic information function in poster creative design [J]. Art Education, 2017, Z6: 219-220. 